\title{
Optimal TAS for Cross-Interference Mitigation in Cognitive MIMO MRC Systems
}

\author{
Zakaria El-Moutaouakkil(1) $^{(1)}$ Kamel Tourki $^{(2)}$, Samir Saoudi ${ }^{(1)}$, Halim Yanikomeroglu ${ }^{(3)}$ \\ ${ }^{(1)}$ IMT Atlantique Bretagne-Pays de la Loire, Lab-STICC, UBL, 29238 Brest, France \\ ${ }^{(2)}$ Mathematical and Algorithmic Sciences Lab, France Research Center, Huawei Technologies Co., France \\ ${ }^{(3)}$ Department of Sytems and Computer Engineering, Carleton University, Ottawa, ON K1S 5B6, Canada \\ Emails: \{z.elmoutaouakkil@ieee.org, kamel.tourki@huawei.com, samir.saoudi@imt-atlantique.fr, halim@sce.carleton.ca\}
}

\begin{abstract}
In a cost-effective implementation, we address the problem of cross-interference in cognitive multiple-input multiple-output (MIMO) spectrum sharing where mutual cochannel interference (CCI) between the primary and secondary systems is of major concern. First, we propose an optimal transmit-antenna selection (TAS) strategy that instead maximizes the received signal-to-interference-plus-noise ratio (SINR) after maximum-ratio combining (MRC). We statistically analyze the combined SINR structure and derive new expressions of its cumulative and probability distribution functions. Interestingly, we accurately show the substantial gains offered by the proposed TAS strategy in terms of the secondary system outage performance compared to the existent state-of-the-art TAS strategies. Finally, our analytical results are validated by simulations and some important insights are also underlined.
\end{abstract}

Index Terms-cognitive MIMO, spectrum sharing, primary system, secondary system, TAS, SINR, MRC, interference constraint, outage performance, Monte-Carlo simulations.

\section{INTRODUCTION}

Cognitive radio provides a set of spectrum policies to efficiently use our spectrum band while meeting the latency, reliability and massive connectivity requirements of future wireless and mobile networks. Among these policies, cognitive spectrum sharing is admitted as a new radio paradigm where secondary unlicensed users are permitted to access the primary licensed user's spectrum without causing severe performance degradation to their quality-of-service (QoS). Practically, an interference constraint is posed on the secondary system to keep its transmit power below a certain acceptable threshold. Furthermore, the secondary system will in return be subject to the primary system co-channel interference. By and large, to access the spectrum medium of the primary users, these two interference constraints render the secondary system transmission performance under difficult scenarios. To alleviate this difficulty, robust and sophisticated transmission schemes should take place to primarily mitigate the mutual interference between the primary and secondary systems.

Several approaches have been proposed in the literature, among which beamforming, space-time precoding and antenna switching/selection figure prominently as MIMOenabled techniques that can efficiently sort out the dilemma of co-existence on the same spectrum band [2], [3], [4]. From a cost-effective implementation perspective, beamforming and space-time precoding require large feedback overhead and several radio-frequency chains compared to TAS as a form of antenna selection diversity scheme. TAS maintains a good tradeoff between complexity, performance and cost, and is attractive in situations where implementing several co-located antennas is infeasible or costly. This was the case in the LTE uplink (Release 8/9). In this work, we consider TAS in its simplest form where only a single transmit antenna resulting in the minimal mutual interference effect in our cognitive MIMO system is selected. At the receiver side, MRC combining is applied as a receive diversity scheme, thereby the adopted technique is referred to as TAS/MRC.

Currently, the proposed TAS strategies are sub-optimal as they do not lead to the maximum received SINR after MRC. Depending on the type of interference constraint whether regulated in a statistical basis or instantaneously, to the best of the authors knowledge, these strategies do not jointly tackle the mutual interference in cognitive spectrum sharing MIMO MRC systems. For instance, the considered TAS in [8], [9] merely maximizes the signal-to-interference-plus-noise ratio (SNR) and not the SINR. Under a statistical interference constraint, the secondary system simply acquires the secondorder statistic of its CCI on the primary system to adapt its transmit power, therefore the proposed TAS in this case has to only deal with CCI from the primary system. Very recently, we have proposed an optimal TAS for cognitive MIMO MRC systems where a statistical interference constraint is imposed on the secondary system [10]. Capitalizing on this work, we herein underline the following contributions:

- We introduce a novel TAS strategy for cognitive MIMO MRC systems where the spectrum sharing interference constraint is rather imposed in an instantaneous basis. Therefore, it efficiently mitigate the inherited crossinterference in our system compared to [8], [9];

- New analytical expressions of the cumulative distribution function (CDF) and probability distribution function (PDF) of the received SINR under the proposed TAS strategy are derived for the first time;

- We validate our findings by simulations while underling some important insights and remarks. 


\section{DESCRIPTION OF THE CONCEPT}

\section{A. System, Channel and Side Information Models}

Our cognitive spectrum sharing setup consists of a secondary transmitter (S-Tx) with $K$ antennas and a receiver (S$\mathrm{Rx}$ ) with $L$ antennas; both nodes share the spectrum band with its legitimate owners, a primary transmitter (P-Tx) and receiver (P-Rx) that are equipped with a single antenna. The frequency-flat fading channels connecting P-Tx with P-Rx, the $k$ th antenna at S-Tx with the $l$ th antenna at S-Rx, P-Tx with the $l$ th antenna at S-Rx, and the $k$ th antenna at S-Tx with PRx are modeled by the coefficients $h^{p \rightarrow p}, h_{l, k}^{s \rightarrow s}, h_{l}^{p \rightarrow s}$, and $h_{k}^{s \rightarrow p}$, respectively, for $k \in\{1, \ldots, K\}$ and $l \in\{1, \ldots, L\}$ wherever applicable. We assume that these coefficients are mutually independent and drawn from a zero-mean circularly symmetric complex Gaussian distribution with non-identical variances. That is, $h^{p \rightarrow p} \sim \mathcal{C N}\left(0, \lambda_{p p}\right), h_{l, k}^{s \rightarrow s} \sim \mathcal{C N}\left(0, \lambda_{s s}\right)$, $h_{l}^{p \rightarrow s} \sim \mathcal{C N}\left(0, \lambda_{p s}\right)$, and $h_{k}^{s \rightarrow p} \sim \mathcal{C N}\left(0, \lambda_{s p}\right)$ for any $k$ and $l$ indices. Furthermore, the variation of these channel coefficients is supposed to be slow (i.e., quasi-static) compared to the transmission rate of S-Tx and P-Tx. Thus, P-Rx can accurately estimate $h_{k}^{s \rightarrow p}$ for $k \in\{1, \ldots, K\}$ before being fed back to S-Tx without errors for the purpose of transmit power adaptation. Also, we assume that S-Rx perfectly estimates $h_{l, k}^{s \rightarrow s}$ and $h_{l}^{p \rightarrow s}$ for $k \in\{1, \ldots, K\}$ and $l \in\{1, \ldots, L\}$ before applying the MRC on the received signal vector and feeding back S-Tx with the transmit-antenna index $k$ to be selected for transmission. In which case, the accuracy of the feedback load of $\left\lceil\log _{2}(K)\right\rceil$ binary bits from S-Rx where $\lceil u\rceil$ refers to the smallest integer greater than or equals $u$ is justified

\section{B. Interference Constraint and Transmit Power Adaptation}

Pertaining to the underlay spectrum sharing paradigm, the primary system imposes an instantaneous interference constraint on the transmit power of S-Tx, $P_{k}$, so as not to severely degrade its transmission performance. Being instantaneously adjusted, it guarantees that the interference originated from STx remains below a certain interference-to-noise ratio threshold $Q$ irrespective of the interference channel realization, $h_{k}^{s \rightarrow p}$ for $k \in\{1, \ldots, K\}$. In which case, the primary system is seen to not tolerating the occurrence of events where $Q$ is surpassed although statistically maintained with a certain probability. As a result, the maximum transmit power that must not be exceeded by $\mathrm{S}-\mathrm{Tx}, P_{k}$, can be expressed as

$$
P_{k}=\min \left\{\frac{Q N_{0}}{\left|h_{k}^{s \rightarrow p}\right|^{2}}, \bar{P}\right\},
$$

where $N_{0}$ is the additive white Gaussian noise (AWGN) at P$\mathrm{Rx}$ and S-Rx and $\bar{P}$ is a practical transmit power limit that cannot be exceeded anyway by S-Tx. The index $k \in\{1, \ldots, K\}$ in (1) refers to the selected antenna for transmission at S-Tx. For the first time, we introduce an optimal TAS that tackles the interference originated from both channels $h_{k}^{s \rightarrow p}$ and $h_{l}^{p \rightarrow s}$ for $k \in\{1, \ldots, K\}$ and $l \in\{1, \ldots, L\}$ referred to as the crossinterference in our cognitive MIMO setup.

\section{Cross-Interference-Aware TAS}

Irrespective of the antenna selection criterion adopted by $\mathrm{S}$ Tx, the received MRC-combined SINR at S-Rx for a selected antenna $k \in\{1, \ldots, K\}$ at $\mathrm{S}-\mathrm{Tx}$ can be expressed as [5], [6]

$$
\gamma_{k}^{s \rightarrow s}=\frac{P_{k} X_{k}}{P_{p} Z_{k}+N_{0}},
$$

where

$$
\begin{gathered}
X_{k}=\sum_{l=0}^{L}\left|h_{l, k}^{s \rightarrow s}\right|^{2}, \\
Z_{k}=\frac{\left|\sum_{l=1}^{L} h_{l, k}^{s \rightarrow s^{*}} h_{l}^{p \rightarrow s}\right|^{2}}{X_{k}},
\end{gathered}
$$

and $P_{p}$ is the transmit power of P-Tx. Kindly note that the index $k$ jointly impacts $P_{k}, X_{k}$ and $Z_{k}$ in (2). In the simple case of $L=1$ [7], $Z_{k}$ in (4) reduces to $Z=\left|h_{1}^{p \rightarrow s}\right|^{2}$ thus impacted by the TAS criterion being used.

- Existent TAS Strategies: Numerous TAS strategies can be adopted to boost the performance of secondary MIMO MRC systems under the primary system co-channel interference. Among these, in [8], the proposed TAS is based on the maximization of the received SNR, i.e., the transmit antenna $*$ at $\mathrm{S}$-Tx is selected according to

$$
\text { * }=\arg \max _{k \in\{1, \ldots, K\}}\left\{P_{k} X_{k}\right\} .
$$

It differs from [9] in the sense that the selection is based on maximizing the ratio of $X_{k}$ to $\left|h_{k}^{s \rightarrow p}\right|^{2}$ in (1). In which case, the best antenna is selected according to

$$
\text { * }=\arg \max _{k \in\{1, \ldots, K\}}\left\{\frac{X_{k}}{\left|h_{k}^{s \rightarrow p}\right|^{2}}\right\} .
$$

The equality between (5) and (6) is only justified when $\bar{P}$ in (1) tends to infinity. However, for practical limited values of $\bar{P}$, (6) can be misleading to non sub-optimal values of the received SINR at S-Rx

- Proposed Cross-Interference-Aware TAS: For the first time, we propose an optimal TAS strategy that takes into account the three components $P_{k}, X_{k}$ and $Z_{k}$ in (2). Importantly, the mutual interference between the primary and secondary systems reflected in $P_{k}$ and $Z_{k}$ is now jointly mitigated. We instead select the index $k$ leading to the highest received SINR as

$$
\text { * = arg } \max _{k \in\{1, \ldots, K\}}\left\{\gamma_{k}^{s \rightarrow s}\right\} .
$$

As a consequence, $P_{*}, X_{*}$ and $Z_{*}$ become correlated random variables with non-trivial probability distribution functions. In the sequel, we carefully investigate the impact our proposed TAS strategy has on the outage performance of our cognitive $L \times K$ MIMO MRC system. We also highlight some important insights compared to the TAS strategies (5) and (6). 


\section{Outage Performance Analysis}

In this section, we derive the outage probability $o p_{s}$ of our cognitive MIMO system when the proposed TAS/MRC in (7) is adopted. Further, we analyze its asymptotic behavior depending on the involved system parameters.

\section{A. CDF of the MRC Output SINR $\gamma_{*}^{s \rightarrow s}$ at S-Rx}

If S-Tx selects its transmit antenna according to (7), the $\mathrm{CDF}$ of the received SINR after MRC is given by

$$
\begin{gathered}
F_{\gamma_{*}^{s \rightarrow s}}(\gamma)= \\
\mathcal{P}\left(\max _{k \in\{1, \ldots, K\}}\left\{\min \left\{\frac{Q N_{0}}{U_{k}}, \bar{P}\right\} \frac{X_{k}}{P_{p} Z_{k}+N_{0}}\right\}<\gamma\right),
\end{gathered}
$$

where $U_{k}=\left|h_{k}^{s \rightarrow p}\right|^{2}$ and $Z_{k}$ for a given $k \in\{1, \ldots, K\}$ are exponential random variables with scale parameters equaling to $\lambda_{s p}$ and $\lambda_{p s}$, respectively. Initially, $Z_{k}$ as in (4) may not appear to be exponentially distributed expect under the special case of $L=1$ for which $Z_{k}$ reduces to $Z_{k}=\left|h_{1}^{p \rightarrow s}\right|^{2}$. In the general case of an arbitrary $L$, it has been demonstrated in [5] that $Z_{k}$ is still an exponential variable. As for $X_{k}$ in (8), it is drawn from a Gamma distribution with shape and scale parameters equal to $L$ and $\lambda_{s s}$, respectively. In the following theorem, we derive an exact expression of the above CDF.

Theorem 1: Under an instantaneous interference constraint, the CDF of the MRC-combined SINR at S-Rx when the proposed optima TAS strategy in (7) is adopted can be expressed as shown in (17) in the top of next page.

Proof: The bottleneck in deriving (8) resides in dealing with the correlation among the per-transmit-antenna received SINRs $\gamma_{k}^{s \rightarrow s}$ for $k \in\{1, \ldots, K\}$ caused by $Z_{k}$ that embodies the same interference channel $h_{l}^{p \rightarrow s}$ for $l \in\{1, \ldots, L\}$. To get rid of the maximum then the minimum operators in (8), we apply the total probability law by conditioning the event inside (8) firstly on $V_{k}=\sum_{l=1}^{L}\left|h_{l}^{p \rightarrow s}\right|^{2}$ which follows a Gamma distribution with shape and scale parameters equal to $L$ and $\lambda_{p s}$, respectively, and secondly on $U_{k}$. The intuition behind introducing $V_{k}$ in $Z_{k}$ and rewriting the latter (4) as

$$
Z_{k}=\frac{V_{k}}{X_{k}} Y_{k}
$$

where $Y_{k}=\left|\sum_{l=1}^{L} h_{l, k}^{s \rightarrow s^{*}} h_{l}^{p \rightarrow s}\right|^{2} / V_{k}$ is because $V_{k}$ is the cause of correlation among the per-antenna received SINRs $\gamma_{k}^{s \rightarrow s}$. Since $U_{k}$ and $V_{k}$ are independent, the CDF in (8) can be rewritten as follows

$$
\begin{aligned}
& F_{\gamma_{*}^{s \rightarrow s}}(\gamma)=\int_{0}^{+\infty}\left[\int_{0}^{\frac{Q N_{0}}{P}} \Psi\left(\frac{Q N_{0}}{\bar{P}}, v, \gamma\right) \frac{e^{-\frac{u}{\lambda_{s p}}}}{\lambda_{s p}} d u\right. \\
& \left.+\int_{\frac{Q N_{0}}{P}}^{+\infty} \Psi(u, v, \gamma) \frac{e^{-\frac{u}{\lambda_{s p}}}}{\lambda_{s p}} d u\right]^{K} \frac{v^{L-1} e^{-\frac{v}{\lambda_{p s}}}}{\lambda_{p s}^{L} \Gamma(L)} d v,
\end{aligned}
$$

where $\Gamma(n)=(n-1)$ ! is the Gamma function whereas the probability function $\Psi(u, v, \gamma)$ for $u \geq Q N_{0} / \bar{P}$ is given by

$$
\begin{aligned}
\Psi(u, v, \gamma) & =\mathcal{P}\left(\frac{X_{k}}{\frac{P_{p}}{N_{0}} \frac{v}{X_{k}} Y_{k} \mid v+1}<\frac{\gamma u}{Q}\right) \\
& =\Psi_{1}(u, \gamma)+\Psi_{2}(u, v, \gamma)
\end{aligned}
$$

respectively. Following a moment generation function-based approach, it can easily be demonstrated that the joint PDF of $X_{k}$ and $Y_{k} \mid v, f_{X_{k}, Y_{k} \mid v}($,$) , is none but the McKay's bivariate$ Gamma distribution [11] given for $0<y<x$ and $L \geq 2$ by

$$
f_{X_{k}^{1}, Y_{k}^{1} \mid v}(x, y)=\frac{(x-y)^{L-2} e^{-\frac{x}{\lambda_{s s}}}}{\lambda_{s s}^{L} \Gamma(L-1)} .
$$

Fortunately, the inequality $0<Y_{k}<X_{k}$ holds true by default given the structure of each of the two variables $X_{k}$ and $Y_{k}$. Otherwise, $f_{X_{k}^{1}, Y_{k}^{1} \mid v}(x, y)$ will be a truncated bivariate distribution. Since (11) involves two random variables, we proceed by defining the region $\mathcal{R}=\left\{\mathcal{R}_{1} \cup \mathcal{R}_{2}\right\}$ over which $f_{X_{k}^{1}, Y_{k}^{1} \mid v}(x, y)$ should be integrated. $\mathcal{R}$ can be deduced from the solution to the inequalities $x^{2} Q N_{0}<\gamma u\left(P_{p} v y+N_{0} x\right)$ and $0<y<x$ as

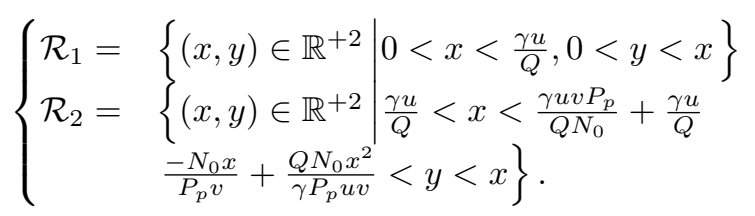

From (12), $\Psi_{1}(u, \gamma)$ in the second line of (11) can be rewritten with the help of $[12,3.351 .1]$ as

$$
\begin{aligned}
\Psi_{1}(u, \gamma) & =\iint_{\mathcal{R}_{1}} \frac{(x-y)^{L-2} e^{-\frac{x}{\lambda_{s s}}}}{\lambda_{s s}^{L} \Gamma(L-1)} d y d x \\
& =\bar{\gamma}\left(L, \frac{\gamma u}{Q \lambda_{s s}}\right)
\end{aligned}
$$

In turn, $\Psi_{2}(u, v, \gamma)$ in (11) can further be developed to a closed-form expression as

$$
\begin{gathered}
\Psi_{2}(u, v, \gamma)=\iint_{\mathcal{R}_{2}} \frac{(x-y)^{L-2} e^{-\frac{x}{\lambda_{s s}}}}{\lambda_{s s}^{L} \Gamma(L-1)}(x, y) d y d x \\
=\frac{(-1)^{L} e^{-\frac{\gamma u}{Q \lambda_{s s}}}}{\Gamma(L)} \sum_{k=0}^{L-1}\left(\begin{array}{c}
L-1 \\
k
\end{array}\right)\left(\frac{Q N_{0} \lambda_{s s}}{\gamma u}\right)^{k} e^{-\frac{\gamma P_{p} u v}{Q N_{0} \lambda_{s s}}} \\
\times \frac{\left(P_{p} v+N_{0}\right)^{L-1-k}}{\left(P_{p} v\right)^{L-1}} \gamma\left(L+k,-\frac{\gamma P_{p} u v}{Q N_{0} \lambda_{s s}}\right)
\end{gathered}
$$

where $\bar{\gamma}(n, x)=\gamma(n, x) / \Gamma(n)=1-e^{-x} \sum_{i=0}^{n-1} x^{i} / i$ ! for an integer $n$ and real $x$ is the regularized lower incomplete Gamma function. From (15) to (16), we made the following change of variable $t=\frac{\gamma u}{Q N_{0}}\left(P_{p} v+N_{0}\right)-x$ before expanding the resulting binomial inside the integral. Finally, we get (16) 


$$
\begin{aligned}
F_{\gamma_{*}^{s \rightarrow s}}(\gamma) & =\int_{0}^{+\infty}\left[\left(1-e^{-\frac{Q N_{0}}{\lambda_{s p} P}}\right)\left(\Psi_{1}\left(\frac{Q N_{0}}{\bar{P}}, \gamma\right)+\Psi_{2}\left(\frac{Q N_{0}}{\bar{P}}, v, \gamma\right)\right)+\Psi_{3}(\gamma)\right. \\
& \left.+\frac{(-1)^{L}}{\Gamma(L)} \sum_{k=0}^{L-1}\left(\begin{array}{c}
L-1 \\
k
\end{array}\right)\left(\frac{Q N_{0} \lambda_{s s}}{\gamma}\right)^{k} \frac{\left(P_{p} v+N_{0}\right)^{L-1-k}}{\left(P_{p} v\right)^{L-1}} \Psi_{4}(v, \gamma)\right]^{K} \frac{v^{L-1} e^{-\frac{v}{\lambda_{p s}}}}{\lambda_{p s}^{L} \Gamma(L)} d v
\end{aligned}
$$

$$
\Psi_{3}(\gamma)=\int_{\frac{Q N_{0}}{P}}^{+\infty} \bar{\gamma}\left(L, \frac{\gamma u}{Q \lambda_{s s}}\right) \frac{e^{-\frac{u}{\lambda_{s p}}}}{\lambda_{s p}} d u=e^{-\frac{Q N_{0}}{P \lambda_{s p}}}-\frac{1}{\lambda_{s p}} \sum_{m=0}^{L-1} \frac{\left(\frac{\gamma}{Q \lambda_{s s}}\right)^{m}}{m !} \frac{\Gamma\left(m+1, \frac{Q N_{0}}{P}\left(\frac{\gamma}{Q \lambda_{s s}}+\frac{1}{\lambda_{s p}}\right)\right)}{\left(\frac{\gamma}{Q \lambda_{s s}}+\frac{1}{\lambda_{s p}}\right)^{m+1}}
$$

$$
\frac{\Psi_{4}(k, v, \gamma)}{\Gamma(L+k)}=\frac{1}{\lambda_{s p}} g\left(-k, \frac{Q N_{0}}{\bar{P}}, \frac{\gamma}{Q \lambda_{s s}}+\frac{\gamma P_{p} v}{Q N_{0} \lambda_{s s}}+\frac{1}{\lambda_{s p}}\right)-\sum_{n=0}^{L+k-1} \frac{\left(\frac{-\gamma P_{p} v}{Q N_{0} \lambda_{s s}}\right)^{n}}{\lambda_{s p} n !} g\left(n-k, \frac{Q N_{0}}{\bar{P}}, \frac{\gamma}{Q \lambda_{s s}}+\frac{1}{\lambda_{s p}}\right)
$$

with the help of [12, 3.351.1]. By substituting (14) and (16) into (10), we end up with (17) where $\Psi_{3}(\gamma)$ in (18) and

$$
\begin{aligned}
\Psi_{4}(k, v, \gamma) & =\frac{1}{\lambda_{s p}} \int_{\frac{Q N_{0}}{P}}^{+\infty} \frac{e^{-u\left(\frac{\gamma}{Q \lambda_{s s}}+\frac{\gamma P_{p} v}{Q N_{0} \lambda_{s s}}+\frac{1}{\lambda_{s p}}\right)}}{u^{k}} \\
& \times \gamma\left(L+k,-\frac{\gamma P_{p} u v}{Q N_{0} \lambda_{s s}}\right) d u,
\end{aligned}
$$

further developed in (19), are both evaluated by expanding the lower incomplete Gamma function and using $[12,3.351 .2$ and 3.351.4]. In (19), the function $g(s, a, b)$ for $a>0, b>0$ and $s \in \mathbb{Z}$ has been introduced as

$$
\begin{aligned}
g(s, a, b) & =\int_{a}^{+\infty} u^{s} e^{-b u} d u \\
& =\left\{\begin{array}{ll}
\frac{\Gamma(s+1, a b)}{b^{s+1}} & ; s \geq 0 \\
(-1)^{-s} \frac{\operatorname{Ei}(-a b)}{(-s-1) !} & ; s=-1 \\
(-1)^{-s} \frac{\operatorname{Ei}(-a b)}{(-s-1) !}+\frac{e^{-a b}}{a^{n}} \times & (-a b)^{r} \\
\sum_{r=0}^{-s-2} \frac{(-s-1)(-s-2) \cdots(-s-1-r)}{(-s)} & ; s \leq-2
\end{array},\right.
\end{aligned}
$$

where $\Gamma(n, x)$ and $\operatorname{Ei}(x)$ for an integer $n$ and real $x$ are the upper incomplete Gamma and exponential integral functions, respectively. The integral in (17) can further be expanded but due to space limitation, we resort to its numerical calculation using mathematical softwares such as MATHEMATICA.

\section{B. PDF of the MRC Output SINR $\gamma_{*}^{s \rightarrow s}$ at $S-R x$}

An important consequence of deriving the CDF in (17) is that it can serve for calculating the PDF of $\gamma_{*}^{s \rightarrow s}$ by derivation as $f_{\gamma_{*}^{s \rightarrow s}}(\gamma)=\partial F_{\gamma_{*}^{s \rightarrow s}}(\gamma) / \partial \gamma$.

Corollary 1: The PDF of the received SINR after MRC, $f_{\gamma_{*}^{s \rightarrow s}}($.$) , when the proposed optimal TAS strategy in (7) is$ used can expressed as (22) in the top of next page.
Proof: Inspired by the derivation roadmap of the CDF $F_{\gamma_{*}^{s \rightarrow s}}($.$) , it follows from (17) that f_{\gamma_{*}^{s} \rightarrow s}(\gamma)$ is given by (22) where $\dot{\Psi}_{1}(u, \gamma)=\frac{\partial}{\partial \gamma} \Psi_{1}(u, \gamma)=$ $\gamma^{L-1} u^{L} e^{-\frac{\gamma u}{Q \lambda_{s s}}} /\left(Q \lambda_{s s}\right)^{L} \Gamma(L)$ and

$$
\begin{gathered}
\dot{\Psi}_{2}(u, v, \gamma)=\frac{\partial}{\partial \gamma} \Psi_{2}(u, v, \gamma)=-\frac{\gamma^{L-1} u^{L} e^{-\frac{\gamma u}{Q \lambda_{s s}}}}{\left(Q \lambda_{s s}\right)^{L} \Gamma(L)}+ \\
\frac{(-1)^{L-1}}{\lambda_{s s} \Gamma(L-1) Q N_{0}} \sum_{k=0}^{L}\left(\begin{array}{l}
L \\
k
\end{array}\right)\left(\frac{Q N_{0} \lambda_{s s}}{\gamma}\right)^{k} \frac{e^{-\frac{\gamma u}{\lambda_{s s} Q}}}{u^{k-1}} e^{-\frac{\gamma P_{p u v}}{\lambda_{s s} Q N_{0}}} \\
\times \frac{\left(P_{p} v+N_{0}\right)^{L-k}}{\left(P_{p} v\right)^{L-1}} \gamma\left(L+k-1,-\frac{P_{p} u v \gamma}{Q N_{0} \lambda_{s s}}\right)
\end{gathered}
$$

while $\dot{\Psi}_{3}(\gamma)=\frac{\partial}{\partial \gamma} \Psi_{3}(\gamma)$ and $\dot{\Psi}_{4}(k, v, \gamma)=\frac{\partial}{\partial \gamma} \Psi_{4}(k, v, \gamma)$ are derived in closed-form expressions in (23) and (24), respectively. After substituting their explicit expressions into (22), we obtain the PDF of $\gamma_{*}^{s \rightarrow s}$ in the form of a single integral similarly to the CDF in (17), thus can accurately be evaluated via numerical integration.

\section{Exact and Asymptotic Outage Performance}

Using (17), the outage probability of our cognitive MIMO system is given by $o p_{s}=F_{\gamma_{*}^{s \rightarrow s}}(\Phi)$ where $\Phi$ is a certain SINR threshold below which the secondary system falls in outage. Without loss of generality, if we assume that $\bar{\rho}=$ $\bar{P} / N_{0}=P_{p} / N_{0}$ then $o p_{s}$ will decrease as $\bar{\rho}$ increase until S-Tx starts transmitting with $Q N_{0} /\left|h_{k}^{s \rightarrow p}\right|^{2}$ according to (1). Meanwhile, P-Tx will continue to pomp an increased amount of interference as $\bar{\rho}$ goes high. Therefore, $o p_{s}$ will climb (goes up) after a certain $\bar{\rho}$ because $Q$ is regarded as a fixed constant despite the primary system operates at high SINR ratios. However, a more flexible instantaneous interference constraint may adapt with $P_{p} / N_{0}$ leading to a proportional $Q$ that increases with $\bar{\rho}$. In which case, $o p_{s}$ will saturate (instead of going worse) at an outage floor $o p F$ due once again to the interference constraint (1) imposed on S-Tx. 


$$
\begin{gathered}
f_{\gamma_{*}^{s \rightarrow s}}(\gamma)=K \int_{0}^{+\infty}\left[\left(1-e^{-\frac{Q N_{0}}{\lambda_{s p} P}}\right)\left(\Psi_{1}\left(\frac{Q N_{0}}{\bar{P}}, \gamma\right)+\Psi_{2}\left(\frac{Q N_{0}}{\bar{P}}, v, \gamma\right)\right)+\Psi_{3}(\gamma)+\frac{(-1)^{L}}{\Gamma(L)} \sum_{k=0}^{L-1}\left(\begin{array}{c}
L-1 \\
k
\end{array}\right)\right. \\
\left.\times\left(\frac{Q N_{0} \lambda_{s s}}{\gamma}\right)^{k} \frac{\left(P_{p} v+N_{0}\right)^{L-1-k}}{\left(P_{p} v\right)^{L-1}} \Psi_{4}(v, \gamma)\right]^{K-1}\left[\left(\left(1-e^{-\frac{Q N_{0}}{\lambda_{s p} P}}\right)\left(\dot{\Psi}_{1}\left(\frac{Q N_{0}}{\bar{P}}, \gamma\right)+\dot{\Psi}_{2}\left(\frac{Q N_{0}}{\bar{P}}, v, \gamma\right)\right)\right)\right. \\
\left.+\dot{\Psi}_{3}(\gamma)+\frac{(-1)^{L}}{\Gamma(L)} \sum_{k=0}^{L-1}\left(\begin{array}{c}
L-1 \\
k
\end{array}\right)\left(\frac{Q N_{0} \lambda_{s s}}{\gamma}\right)^{k} \frac{\left(P_{p} v+N_{0}\right)^{L-1-k}}{\left(P_{p} v\right)^{L-1}}\left(\dot{\Psi}_{4}(k, v, \gamma)-\frac{k \Psi_{4}(k, v, \gamma)}{\gamma}\right)\right] \frac{v^{L-1} e^{-\frac{v}{\lambda_{p s}}}}{\lambda_{p s}^{L} \Gamma(L)} d v .
\end{gathered}
$$

$$
\dot{\Psi}_{3}(\gamma)=\frac{1}{\lambda_{s p}} \frac{\gamma^{L}}{\left(Q \lambda_{s s}\right)^{L-1} \Gamma(L)} \int_{\frac{Q N_{0}}{P}}^{+\infty} u^{L-1} e^{-u\left(\frac{\gamma}{Q \lambda_{s s}}+\frac{1}{\lambda_{s p}}\right)} d u=\frac{1}{\lambda_{s p}} \frac{\gamma^{L} Q \lambda_{s s}}{\Gamma(L)} \frac{\Gamma\left(L, \frac{Q N_{0}}{P}\left(\frac{\gamma}{Q \lambda_{s s}}+\frac{1}{\lambda_{s p}}\right)\right)}{\left(\gamma+\frac{Q \lambda_{s s}}{\lambda_{s p}}\right)^{L}} .
$$

$$
\Psi_{4}(k, v, \gamma)=-\left(\frac{1}{Q \lambda_{s s}}+\frac{P_{p} v}{Q N_{0} \lambda_{s s}}\right) \Psi_{4}(k-1, v, \gamma)+\frac{\gamma^{L+k}}{\lambda_{s p}} \frac{\left(-\frac{P_{p} v}{Q N_{0} \lambda_{s s}}\right)^{L+k-1}}{\left(\frac{\gamma}{Q \lambda_{s s}}+\frac{1}{\lambda_{s p}}\right)^{L}} \Gamma\left(L, \frac{Q N_{0}}{\bar{P}}\left(\frac{\gamma}{Q \lambda_{s s}}+\frac{1}{\lambda_{s p}}\right)\right) .
$$
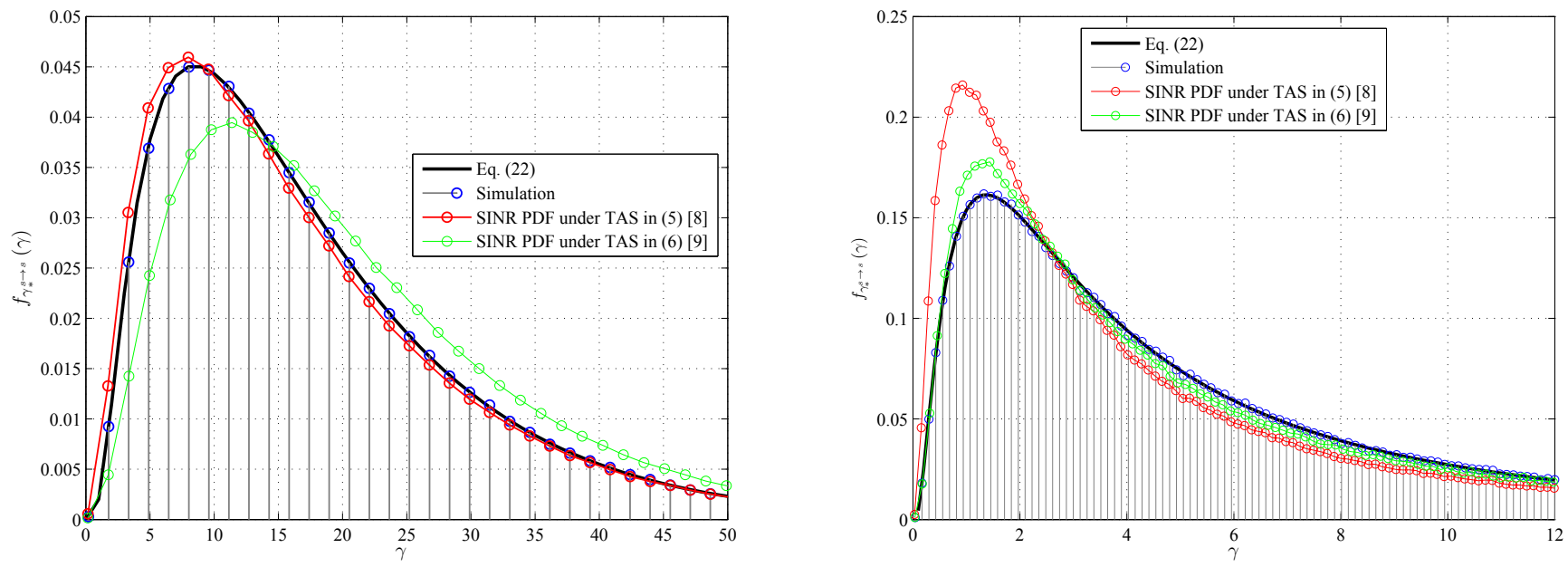

Figure 1. Comparison between the PDF of the received SINRs when our proposed optimal TAS and the ones adopted in [8] and [9] are considered. The simulations are carried out under fixed system settings yet the figure on the left (a) is generated for $\lambda_{p s}=0.1$ whereas that on the right (b) is obtained for $\lambda_{p s}=1$.

\section{Simulation Results}

To validate the correctness of our analytical results, we double-check (22) by simulation and compare it with the one obtained under the TAS strategies in (5) and (6). Then, we move on to analyze and compare the outage probability of our cognitive MIMO system when the TAS strategies under focus are used.

\section{A. PDF of the Received SINR under (5), (6) and (7)}

We generate the PDF of $\gamma_{*}^{s \rightarrow s}$ by simulation and compare it with our derived expression in (22) under fixed system settings $K=L=3, \lambda_{s s}=\lambda_{s p}=1, Q=5 \mathrm{~dB}, \bar{\rho}=10 \mathrm{~dB}$ and variant value of $\lambda_{p s} \in\{0.1,1\}$. On the one hand, this shows the exactness of our mathematical approach and followed derivation steps in the previous section. On the other hand, $f_{\gamma_{s}^{s} \rightarrow s}(\gamma)$ is seen to approach the PDF of the received SINR when the TAS strategy (5) is being adopted [8] for low values of $\lambda_{p s}$. Equivalently, we are weakening the impact of the interference originated by P-Tx on S-Rx by taking $\lambda_{p s} \rightarrow 0$, hence our proposed optimal TAS strategy in (7) tends to coincide with (5) in lower values than $\lambda_{p s}=0.1$ as shown in Fig. 1-(a). In general, the PDF of the received SINRs got clearly separated as $\lambda_{p s}$ goes higher as shown in Fig. 1-(b) for $\lambda_{p s}=1$. For any arbitrary cognitive MIMO system settings, the proposed TAS strategy manifests the optimal results either in terms of the outage or capacity performance. 

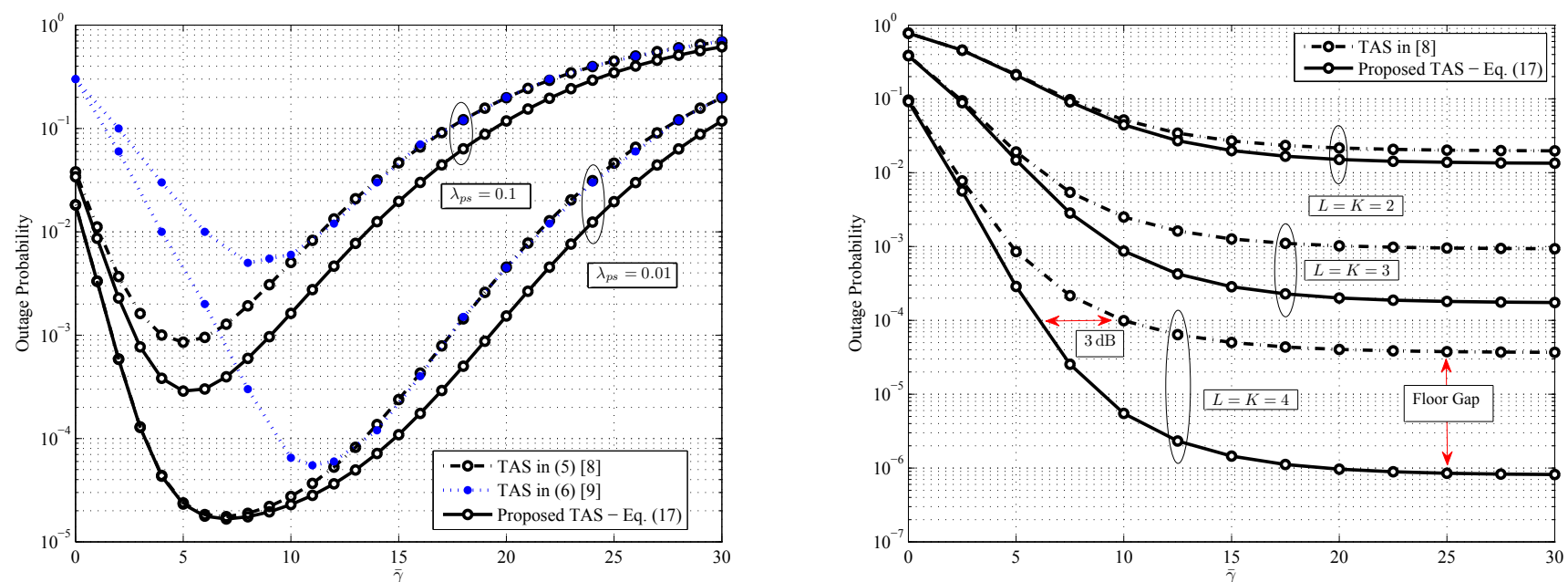

Figure 2. The secondary system outage probability of our cognitive MIMO MRC system with TAS in (7) under different system settings. On the left (a), the curves are obtained under a fixed $Q=5 \mathrm{~dB}$ while those on the right (b) are obtained under an adaptive $Q=\bar{\rho}$.

\section{B. Optimal Outage Performance Results}

The analytical expression of the outage probability $o p_{s}=$ $F_{\gamma_{x}^{s \rightarrow s}}(\Phi)$ derived in (17) where $\Phi=2^{\mathcal{R}}-1$ and $\mathcal{R}=2$ is depicted in solid line in both figures Fig. 2-(a) and Fig. 2-(b) against the dotdashed and dashed ones resulting from the use of the TAS strategies in [8] and [9], respectively. In Fig. 2-(a), our simulations are conducted for $K=L=4, \lambda_{s s}=\lambda_{s p}=1$, and $\lambda_{p s}=0.1$ versus $\bar{\rho}=\bar{P} / N_{0}=P_{p} / N_{0}$ for example and fair comparison. These settings can arbitrary be modified. Apparently, the optimality of the obtained results under the proposed TAS strategy over [8] and [9] is quite clear over the entire range of $\bar{\rho}$. We confirm our earlier observation saying that similitude between (5) and (6) is only justified for high values of $\bar{\rho}$, typically greater than $15 \mathrm{~dB}$. Otherwise, for low values of $\bar{\rho}$, (5) remains a sub-optimal TAS strategy compared to (6) while providing a good complexity-performance tradeoff compared to (7). When $Q$ grows proportionally with $\bar{\rho}$ as in Fig. 2-(b), relaxation of the interference constraint posed on the transmit power of S-Tx results in $o p_{s}$ converges to an outage floor $o p_{s}=o p F_{s}$ despite $\bar{\rho}$ continues to grow. This confirms our discussion in Subsection III-C, and shows the substantial gains offered by our optimal TAS whether we operate at high (floor gap gets increasingly pronounced for loaded MIMO systems) or low (a gain of $3 \mathrm{~dB}$ is observed at $\bar{\rho}=7 \mathrm{~dB}$ ) values of $\bar{\rho}$. Now that the mutual interference effect is minimized by the proposed TAS strategy, the secondary system can be equipped with advanced decoding schemes that can even suppress the residual CCI effect therefore turning $o p F_{s}$ to decay down as $\bar{\rho}$ increases.

\section{CONClusion}

In this contribution, we introduced a novel and optimal TAS strategy for cognitive spectrum sharing MIMO MRC systems. Also, we have derived analytical expressions of the CDF and PDF of the received SINR under the introduced TAS strategy.
Our numerical and simulation results reveals the exactness of the followed mathematical derivations roadmap as well as accuracy and optimality of the obtained results.

\section{REFERENCES}

[1] P. Kolodzy, "Spectrum policy task force," Federal Comm. Commission, Washington, DC, USA, Tech. Rep. ET Docket no. 02-135, Nov. 2002.

[2] K. Huang and R. Zhang, "Cooperative feedback for multi-antenna cognitive radio networks," IEEE Trans. on Signal Processing, vol. 59, no. 2, pp. 747-758, Feb. 2011.

[3] J. Lui, W. Chen, Z. Cao, and Y. J. Zhang, "Cooperative beamforming for cognitive radio networks: A cross-layer design," IEEE Trans. on Wireless Communications., vol. 60, no. 5, pp. 1420-1431, May 2012.

[4] L. Sboui, H. Ghazzai, Z. Rezki, and M. S. Alouini, "Precoder design and power allocation for MIMO cognitive radio two-way relaying systems," IEEE Trans. on Communications, vol. 64, no. 10, pp. 4111-4120, Oct. 2016.

[5] A. Shah and A. M. Haimovich, "Performance analysis of maximal ratio combining and comparison with optimum combining for mobile radio communications with cochannel interference," IEEE Trans. on Vehicular Technology, vol. 49, no. 4, pp. 1417-1425, Aug. 2004.

[6] M. Kang and M.-S. Alouini, "A comparative study on the performance of MIMO MRC systems with and without cochannel interference," IEEE Trans. Communications, vol. 52, no. 8, pp. 1417-1425, Aug. 2004.

[7] M. Hanif, H. C. Yang, and M. S. Alouini, "Transmit Antenna selection for power adaptive underlay cognitive radio with instantaneous interference constraint," IEEE Trans. on Communications, vol. 65, no. 6, pp. 2357-2367, June 2017.

[8] F. A. Khan, K. Tourki, M. S. Alouini, and K. A. Qaraqe, "Performance analysis of a power limited spectrum sharing system with TAS/MRC," IEEE Trans. on Signal Processing, vol. 62, no. 4, pp. 954-967, Feb.15, 2014.

[9] K. Tourki, F. A. Khan, K. A. Qaraqe, H. C. Yang, and M. S. Alouini, "Exact performance analysis of MIMO cognitive radio systems using transmit antenna selection," IEEE Journal on Selected Areas in Communications, vol. 32, no. 3, pp. 425-438, March 2014.

[10] Z. El-Moutaouakkil, K. Tourki, H. Yanikomeroglu, and S. Saoudi, "TAS strategies for incremental cognitive MIMO relaying: New results and accurate comparison", IEEE Access, vol. 6, pp. 23480-23499, 2018.

[11] A. T. McKay, "Sampling from batches," Journal of the Royal Statistical Society-Supplement 1, 1934, 207-216.

[12] I. S. Gradshteyn and I. M. Ryzhik, Table of Integrals, Series, and Products, 7th ed. San Diego, CA: Academic, 2007. 